\title{
Phase II study of weekly oxaliplatin plus infusional fluorouracil and folinic acid (FUFOX regimen) as first-line treatment in metastatic
} gastric cancer

\section{F Lordick ${ }^{*}$, , S Lorenzen', J Stollfuss', U Vehling-Kaiser², F Kullmann ${ }^{3}$, M Hentrich ${ }^{4}$, R Zumschlinge ${ }^{5}$, H Dietzfelbinger', J Thoedtmann', M Hennig', T Seroneit', R Bredenkamp ${ }^{8}$, J Duyster' and C Peschel'}

'Clinic Rechts der Isar, Technical University of Munich, Ismaninger Str. 22, 81675 Munich, Germany; ${ }^{2}$ Oncological Outpatient Clinic, Heilig-Geist-Gasse 4I I, 84028 Landshut, Germany; ${ }^{3}$ University Clinic of Regensburg, Franz-Josef-Strauss-Allee II, 93053 Regensburg, Germany; ${ }^{4}$ Clinic Harlaching, Sanatoriumsplatz 2, 81545 Munich, Germany; ${ }^{5}$ Clinic Traunstein, Cuno-Niggl-Str. 3, 83278 Traunstein, Germany; ${ }^{6}$ Schindlbeck-Clinic, Seestr. 43, 82211 Herrsching, Germany; ${ }^{7}$ Sanofi-Aventis Group, Potsdamer Str. 8, 10785 Berlin, Germany; ${ }^{8}$ Center for Clinical Studies, Ismaninger Str. 22, 81675 Munich, Germany

Oxaliplatin plus fluorouracil/folinic acid (5-FU/FA) every 2 weeks has shown promising activity in advanced gastric cancer. This study assessed the efficacy and safety of weekly oxaliplatin plus 5-FU/FA (FUFOX regimen) in the metastatic setting. Patients with previously untreated metastatic gastric cancer received oxaliplatin $\left(50 \mathrm{mg} \mathrm{m}^{-2}\right)$ plus FA $\left(500 \mathrm{mg} \mathrm{m}^{-2}\right.$, 2-h infusion) followed by 5 -FU (2000 $\mathrm{mg} \mathrm{m}^{-2}, 24-\mathrm{h}$ infusion) given on days I, 8, 15 and 22 of a 5-week cycle. The primary end point of this multicentre phase II study was the response rate according to RECIST criteria. A total of 48 patients were enrolled. Median age was 62 years and all patients had metastatic disease, with a median number of three involved organs. The most common treatment-related grade $3 / 4$ adverse events were diarrhoea (17\%), deep vein thrombosis (15\%), neutropenia (8\%), nausea (6\%), febrile neutropenia (4\%), fatigue (4\%), anaemia (4\%), tumour bleeding (4\%), emesis (2\%), cardiac ischaemia (2\%) and pneumonia (2\%). Grade I/2 sensory neuropathy occurred in $67 \%$ of patients but there were no episodes of grade 3 neuropathy. Intent-to-treat analysis showed a response rate of $54 \%$ (95\% Cl, 39-69\%), including two complete responses. At a median follow-up of 18.1 months (range $11.2-26.2$ months), median survival is 11.4 months $(95 \% \mathrm{Cl}, 8.0-14.9$ months) and the median time to progression is 6.5 months (95\% Cl, 3.9-9.2 months). The weekly FUFOX regimen is well tolerated and shows notable activity as first-line treatment in metastatic gastric cancer. British Journal of Cancer (2005) 93, 190-194. doi:10.1038/sj.bjc.6602697 www.bjcancer.com

Published online 12 July 2005

(c) 2005 Cancer Research UK

Keywords: oxaliplatin; fluorouracil; metastatic gastric cancer; first-line

Systemic chemotherapy has been shown to prolong survival and to relieve symptoms in advanced gastric cancer (Glimelius et al, 1997). However, the number of patients who benefit from treatment is still limited and the optimal chemotherapy regimen has not yet been defined. Platinum-free regimens exhibited disappointing efficacy in randomised trials. Cisplatin-based regimens, for example the combination of epirubicin, cisplatin, and 5-FU (ECF), have demonstrated superior efficacy, albeit with significant toxicity and inconvenience from the patients' perspective (Waters et al, 1999; Vanhoefer et al, 2000).

Oxaliplatin is a third-generation diaminocyclohexane platinum compound proven in numerous clinical trials to be active in various tumour types. In advanced colorectal cancer, treatment with oxaliplatin plus 5-fluorouracil/folinic acid (5-FU/FA) led to significantly increased overall survival and progression-free survival compared with former standard regimens (de Gramont et al, 2000; Giacchetti et al, 2000; Goldberg et al, 2004). Therefore, in many countries this combination forms the new standard of

*Correspondence: Dr F Lordick; E-mail: f.lordick@Irz.tu-muenchen.de Received 27 April 2005; revised 8 June 2005; accepted 9 June 2005; published online 12 July 2005 care in the first-line treatment of metastatic disease. In advanced gastric cancer, biweekly oxaliplatin plus 5-FU/FA has shown considerable antitumour activity in phase II trials (Louvet et al, 2002a; Al-Batran et al, 2004). Oxaliplatin shows a better toxicity profile than cisplatin. Its main and dose-limiting toxicity is acute, cumulative peripheral sensory neurotoxicity, resulting in acral paresthaesia and dysesthaesia, which are exacerbated by cold. The weekly application of oxaliplatin plus infusional 5-FU/FA (FUFOX), as proposed by the Arbeitsgemeinschaft Internistische Onkologie (AIO), has proven to be highly effective in first-line metastatic colorectal cancer. The AIO FUFOX regimen is also associated with an acceptable toxicity profile, with a particularly low rate of sensory neuropathy (Grothey et al, 2002).

This phase II trial was designed to assess the efficacy and safety of the weekly combination of oxaliplatin plus infusional 5-FU/FA (FUFOX) in first-line metastatic gastric cancer.

\section{METHODS}

\section{Patient population}

To be eligible for this study, patients had to have histologically confirmed metastatic adenocarcinoma of the stomach or 
oesophagogastric junction. At least one measurable lesion previously untreated with radiotherapy had to be present. No prior treatment for advanced disease was allowed. Patients were allowed to have received prior adjuvant or neoadjuvant chemotherapy/chemoradiation, providing that treatment had been completed $\geqslant 6$ months before inclusion in the study. All patients were $>18$ years of age with an ECOG performance status $\leqslant 2$ and a life expectancy of $\geqslant 12$ weeks.

Patients were required to have adequate bone marrow, renal and hepatic function, defined as an absolute neutrophil count (ANC) $\geqslant 1500 \mu \mathrm{l}$, platelets $\geqslant 100000 \mu \mathrm{l}$, $S$-creatinine $<1.5 \times$ upper limit of normal (ULN), total bilirubin $\leqslant 1.5 \times$ ULN, SGOT and/or SGPT $\leqslant 1.5 \times$ ULN $(\leqslant 5.0 \times$ ULN in the presence of liver metastasis). Patients with any central nervous system metastases or neuropathy $\geqslant$ grade 2 were excluded.

Pretreatment evaluation included signed written informed consent, complete history and physical examination, laboratory tests, CT scans of all areas affected by the tumour and an ECG. A urine or serum pregnancy test was performed in female patients of child-bearing age. All patients gave written informed consent before enrolment and the study was approved by the ethics committee for human research at the Technical University of Munich. The study conformed to the principles of the Declaration of Helsinki and its subsequent amendments.

\section{Treatment plan}

Oxaliplatin $\left(50 \mathrm{mg} \mathrm{m}^{-2}\right)$ was administered simultaneously with FA $\left(500 \mathrm{mg} \mathrm{m}^{-2}\right)$ as a 2-h intravenous infusion, followed immediately by 5 -FU $\left(2000 \mathrm{mg} \mathrm{m}^{-2}\right)$ as a continuous infusion over $24 \mathrm{~h}$. Treatment was repeated every week. One cycle of treatment was defined as 4 weeks of treatment followed by 1 week of rest. In order to avoid severe neurotoxicity, administration of oxaliplatin was limited to every other week in patients receiving more than four cycles. Patients were treated until best response or until there was evidence of disease progression. Patients going off study were allowed to receive any second-line treatment as determined appropriate by their oncologist.

\section{Study evaluations}

A baseline CT was performed within 2 weeks prior to study inclusion. After every second cycle of therapy, patients underwent follow-up CT scans for assessment of response according to RECIST criteria (Therasse et al, 2000). In cases where treatment was discontinued before tumour progression, CT scans were repeated every 3 months. Responses were to be confirmed within 4 weeks and were reviewed centrally by one independent radiologist (J Stollfuss). Patients were considered evaluable for response if they had received at least two cycles (10 weeks) of treatment, with at least one follow-up tumour assessment. Nonevaluable patients were included into the intention-to-treat analyses but reported as being not evaluable.

Patients were monitored every week for laboratory parameters and adverse events. With the exception of peripheral sensory neuropathy, all adverse events were graded using the National Cancer Institute Common Toxicity Criteria (NCI-CTC, version 2.0). Peripheral sensory neuropathy was graded according to a modified oxaliplatin-specific scale (Caussanel et al, 1990): grade 1 - paresthaesias/dysesthaesias of short duration with complete recovery before the next cycle; grade 2 - paresthaesias/dysesthaesias persisting between two cycles without functional impairment; grade 3 - permanent paresthaesias/dysesthaesias resulting in functional impairment. Patients going off study for reasons other than disease progression were evaluated every 3 months during follow-up visits.

\section{Statistical considerations}

The primary end point of this investigator-initiated, multicentre, nonrandomised, open-label, phase II study was to determine the proportion of patients responding to weekly FUFOX. The required number of patients for this trial was calculated according to the Simon two-stage design (Machin and Campbell, 1997), assuming a minimal response rate $\left(\pi_{0}\right)$ of $30 \%$ and a worthwhile-to-detectresponse-rate $\left(\pi_{1}\right)$ of at least $50 \%$. With a power of $80 \%$ and a significance level of $5 \%$ for testing the hypothesis $\mathrm{H}_{0}: \pi \leq \pi_{0} v s \mathrm{H}_{1}$ : $\pi \geqslant \pi_{1}$, this resulted in a sample size of 15 for the first stage. If more than five out of 15 were observed, another 31 patients were to be recruited in the second stage. The drug combination was to be rejected if less than 19 out of 46 patients were observed.

All eligible patients were included in the response, safety and survival analyses. Time to progression (TTP: time from study entry until documented tumour progression) and overall survival (OS: time from study entry until death) were analysed according to the Kaplan-Meier method, and were updated to 15 October 2004. Statistical computations were performed using SPSS (version 12.0).

\section{RESULTS}

\section{Patient characteristics}

A total of 48 patients were enrolled at nine study sites between August 2002 and November 2003. Patient baseline characteristics are listed in Table 1. All patients had metastatic disease, with the liver, lymph nodes, peritoneum and lung being the predominant sites of metastases. The median number of involved organs was three (range $0-5)$. In total, $37(77 \%)$ patients had newly diagnosed

Table I Patient baseline characteristics

\begin{tabular}{lc}
\hline Characteristics & \\
\hline Total number of patients & 48 \\
Median age (range) & $62(41-75)$ years \\
Gender & \\
Male & $39(81 \%)$ \\
Female & $9(19 \%)$ \\
& \\
ECOG performance status & $23(48 \%)$ \\
0 & $24(50 \%)$ \\
I & $1(2 \%)$ \\
2 & \\
Disease status & $37(77 \%)$ \\
Newly diagnosed & $11(23 \%)$ \\
Recurrent & $0(0 \%)$ \\
Locally advanced & $48(100 \%)$ \\
Metastatic & \\
Localisation of the primary tumour & $23(48 \%)$ \\
Stomach & $23(48 \%)$ \\
Oesophagogastric junction & $2(4 \%)$ \\
Gastric stump & \\
Sites of disease & $30(63 \%)$ \\
Liver & $29(60 \%)$ \\
Lymph nodes & $16(33 \%)$ \\
Peritoneum & $13(27 \%)$ \\
Lung & $5(10 \%)$ \\
Oone & $6(12 \%)$ \\
\hline Others & \\
\hline
\end{tabular}

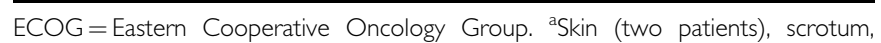
adrenals, spleen and kidney. 
primary metastatic disease. In all of those patients, the primary tumour had been left in situ whereas 11 (23\%) patients had recurrent disease after previous surgery intending to cure the primary.

\section{Feasibility and safety}

The median number of completed cycles was 4 (range $0-6$ cycles). Adherence to the planned doses of both oxaliplatin and 5-FU was high. Dose reductions of one or both agents to $<80 \%$ of initial doses were required in $<10 \%$ of patients. The median administered cumulative dose of oxaliplatin was $800 \mathrm{mg} \mathrm{m}^{-2}$.

All grade 1/2 treatment-related adverse events observed in more than $5 \%$ of patients are listed in Table 2 . While it affected $67 \%$ of patients, sensory neuropathy was generally mild. All grade 3/4 treatment-related adverse events are listed in Table 2 . The most common grade $3 / 4$ events (affecting $>5 \%$ of patients) were diarrhoea, deep vein thrombosis, neutropenia and nausea. Of note, stomatitis and sensory neuropathy $>$ grade 2 were not reported in any of the patients.

Serious adverse events (SAEs) were reported in 14 patients. Two SAEs, one case of septic diarrhoea with neutropenia and one of stroke were fatal. Both deaths occurred during the first two treatment cycles. As there were no other early deaths within the first 2 months after study entry, the 60-day mortality rate was $4.2 \%$.

\section{Efficacy}

Tumour response was evaluable according to RECIST criteria in 45 patients. Three (6\%) patients who did not undergo the first follow-up tumour assessment due to early death or premature termination of therapy (patient preference) were included in the intention-to-treat analyses as nonevaluable subjects. There were two (4\%) complete responses and $24(50 \%)$ patients achieved a partial remission, resulting in an overall response rate of $54 \%$ (95\% CI, 39-69\%). Eight (17\%) patients had stable disease and $11(23 \%)$ had progressive disease at the first follow-up tumour assessment.

A total of $25(52 \%)$ patients received second- and third-line chemotherapies: 13 irinotecan-based, seven taxane-based, three platinum-based, one epirubicin, one etoposide, and four investigational drugs. Two patients underwent secondary palliative

Table 2 Treatment-related adverse events

\begin{tabular}{lcc}
\hline & \multicolumn{2}{c}{ No. of patients (\%) } \\
\cline { 2 - 3 } Event & Grade I/2 & Grade 3/4 \\
\hline Anaemia & $21(44 \%)$ & $2(4 \%)$ \\
Neutropenia & $6(13 \%)$ & $4(8 \%)$ \\
Febrile neutropenia & $\mathrm{NA}$ & $2(4 \%)$ \\
Thrombocytopenia & $7(15 \%)$ & $1(2 \%)$ \\
Diarrhoea & $17(35 \%)$ & $8(17 \%)$ \\
Constipation & $4(8 \%)$ & $0(0 \%)$ \\
Stomatitis & $7(15 \%)$ & $0(0 \%)$ \\
Nausea & $33(69 \%)$ & $3(6 \%)$ \\
Emesis & $13(27 \%)$ & $1(2 \%)$ \\
Fatigue & $21(44 \%)$ & $2(4 \%)$ \\
Deep vein thrombosis & $\mathrm{NA}$ & $7(15 \%)$ \\
Tumour bleeding & $\mathrm{NA}$ & $2(4 \%)$ \\
Cardiac ischaemia & $\mathrm{NA}$ & $1(2 \%)$ \\
Pneumonia & $\mathrm{NA}$ & $1(2 \%)$ \\
Fever & $4(8 \%)$ & $0(0 \%)$ \\
Alopecia & $7(15 \%)$ & $\mathrm{NA}$ \\
Sensory neuropathy & $32(67 \%)$ & $0(0 \%)$ \\
\hline NA & &
\end{tabular}

$\mathrm{NA}=$ not applicable. surgery and one patient was treated with external beam radiation therapy.

Median OS was 11.4 months (95\% CI, 8.0-14.9 months, Figure 1). Median TTP was 6.5 months (95\% CI, 3.9-9.2 months, Figure 2). Median follow-up is 18.1 months (range 11.2-26.2 months).

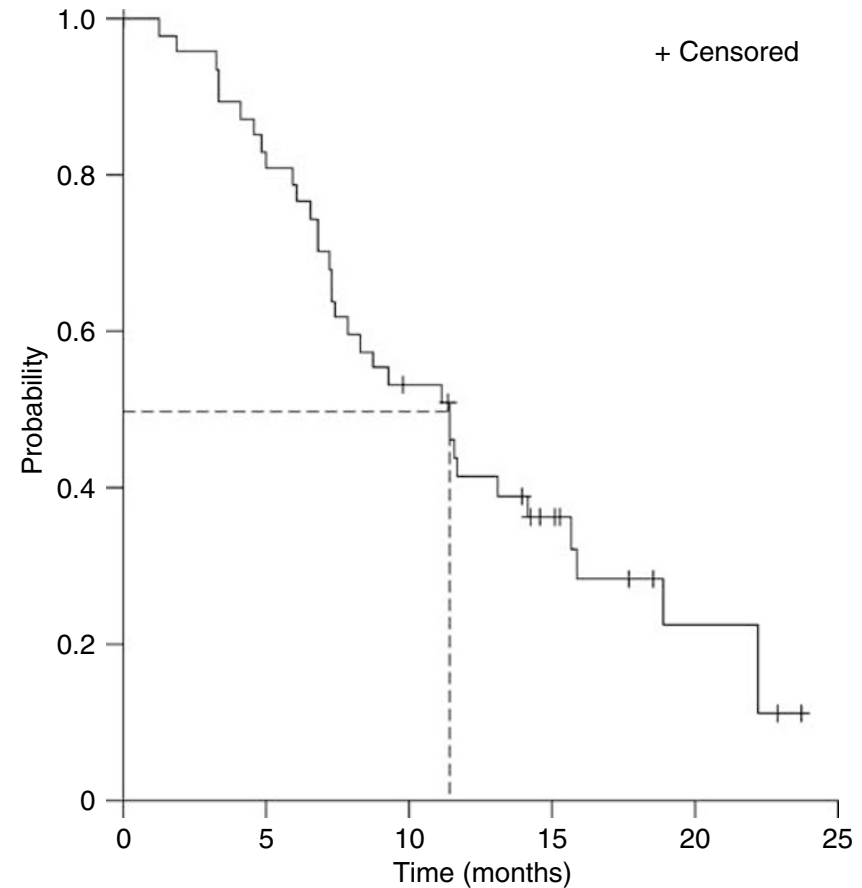

Figure I Kaplan-Meier curve for overall survival in all patients $(n=48)$

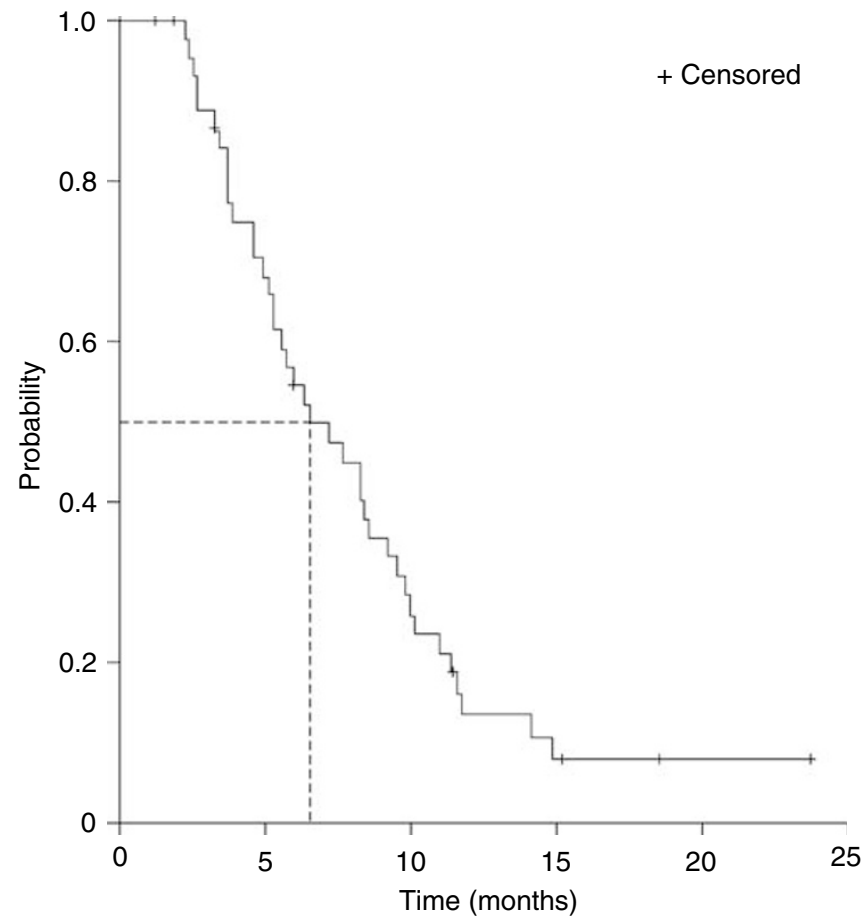

Figure 2 Kaplan-Meier curve for time to progression in all patients $(n=48)$. 


\section{DISCUSSION}

Oxaliplatin combinations have become the mainstay of systemic treatment for advanced gastrointestinal tumours. As a result of the data from randomised phase III trials showing superiority over former standard regimens (de Gramont et al, 2000; Giacchetti et al, 2000; André et al, 2004a; Goldberg et al, 2004), oxaliplatin plus 5-FU/FA has been approved in many countries for the palliative treatment of stage IV colorectal cancer and the adjuvant therapy of stage III colon cancer. Moreover, there is increasing evidence from phase II studies that oxaliplatin combinations have significant activity in other gastrointestinal tumours such as pancreatic cancer (Louvet et al, 2002b; Ducreux et al, 2004) and biliary tract adenocarcinoma (André et al, 2004b). In combination with radiation therapy, oxaliplatin and fluoropyrimidines result in promising tumour remissions in locally advanced oesophageal and rectal cancer (Freyer et al, 2001; Khushalani et al, 2002; Rödel et al, 2003).

In advanced gastric cancer, two previously published phase II studies have shown consistent results regarding the activity of biweekly oxaliplatin-5-FU/FA combinations. These regimens induced objective tumour responses in 43 and $45 \%$ of patients, respectively, and were associated with a median survival of 9.6 and 8.6 months, respectively (Louvet et al, 2002a; Al-Batran et al, 2004). A study from Taiwan assessed the activity of oxaliplatin-5FU/FA given on days 1 and 8, repeated every 3 weeks. This regimen induced an objective tumour reponse in $56 \%$ of the evaluable patients. The median TTP and survival were 5.2 and 10.0 months, respectively (Chao et al, 2004). Although data from phase III trials have not yet been presented, in daily practice the combination of oxaliplatin plus 5-FU/FA is increasingly used by many oncologists for the treatment of advanced gastric cancer.

The objective of this study was to assess the efficacy of weekly oxaliplatin plus 5-FU/FA given 4 out of 5 weeks (FUFOX regimen) as first-line treatment for metastatic gastric and oesophagogastric adenocarcinoma. Leading to an objective response rate of $54 \%$, the activity of FUFOX exceeded the expectations of the trial. This unexpectedly high activity of FUFOX seems to translate into a meaningful clinical benefit, as indicated by the median survival time of 11.4 months.

One might assume that these promising efficacy results are attributable to a patient selection bias. To encounter this wellknown phenomenon in phase II testing, selected study centres included two university hospitals, two community hospitals and five private practices. This represents the typical treatment facilities for cancer patients in Germany. As in three studies on oxaliplatin plus 5-FU/FA in gastric cancer previously published by the French group (Louvet et al, 2002a), the Taiwan group (Chao et al, 2004) and the Frankfurt group (Al-Batran et al, 2004), the majority of patients in our study presented with a good performance status and the median age of 62 years was in the same range as in the three previous trials. Of note, all patients included in our study presented with metastatic disease. Consequently, none of them underwent secondary curative treatment. In contrast, the French group reported on seven of $54(13 \%)$ patients with locally advanced disease and on eight (15\%) patients undergoing complementary surgery or chemoradiation with curative intent.

The three other mentioned studies do not report on the frequency of second-line chemotherapy in their study populations. In our study, the use of second- and even third-line therapies was relatively frequent ( $>50 \%$ of patients). The chosen regimens most frequently included irinotecan or taxanes. Although no phase III trial has yet defined the clinical benefit of second-line chemotherapy in gastric cancer, there is no doubt that second-line treatment with the most recently available agents may be effective in a considerable number of patients. Evidence from phase II trials suggests that irinotecan-containing regimens may be particularly active in this setting (Ajani et al, 2002; Assersohn et al, 2004). Therefore, it is important to acknowledge that the use of secondline chemotherapies may have positively influenced the survival time in our study population.

Treatment compliance for the FUFOX regimen was very good with a dose adherence to both oxaliplatin and 5-FU of $>90 \%$. As already observed by the Taiwan and even more by the Frankfurt group, avoiding the 5-FU bolus administration has led to a dramatic reduction of haematological toxicity. In the French regimen, which included a 5-FU bolus on day 1 , the rate of grade 3 / 4 neutropenia was $38 \%$. This was associated with an $11 \%$ rate of febrile neutropenia. Thus, in our study using weekly oxaliplatin/5FU as well as in the modified version of biweekly FOLFOX as used by the Frankfurt group, bolus 5-FU was omitted. Consequently, the rate of grade $3 / 4$ neutropenia was below $10 \%$ and febrile neutropenia occurred in less than $5 \%$ of the patients in both studies. Compared with the other oxaliplatin/5-FU regimens studied in gastric cancer, the rate of severe diarrhoea was relatively high $(17 \%)$ in our study. However, this is comparable with recently reported findings with the FUFOX regimen in metastatic colorectal cancer, where grade $3 / 4$ diarrhoea affected $21 \%$ of patients (Grothey et al, 2002). It would be worthwhile to investigate whether administering a lower dose of FA, which can itself cause diarrhoea, would reduce this rate.

Unfortunately, in our study, one patient died as a result of septic diarrhoea and neutropenia. The investigators considered this death to be treatment-related. However, the relationship between stroke and death in another patient was judged to be uncertain by the investigators. Nevertheless, these events highlight the importance of careful monitoring of these highly vulnerable patients, even when a generally well-tolerated chemotherapy regimen is administered.

As expected with oxaliplatin-containing regimens, neurotoxicity affected the majority of patients. However, in contrast to studies with the FOLFOX-6 regimen, where $21 \%$ of patients reported functional impairment caused by sensory neuropathy with a median administered oxaliplatin dose of $900 \mathrm{mg} \mathrm{m}^{-2}$ (Louvet et al, 2002a), the intensity of neuropathy observed in our trial, where the median dose of oxaliplatin was $800 \mathrm{mg} \mathrm{m}^{-2}$, did not exceed mild to moderate grades. This observation corresponds well with the recently reported low rate of severe neuropathy with the weekly FUFOX regimen in stage IV colorectal cancer (Grothey et al, 2002). These observations indicate that weekly application of lower doses of oxaliplatin may have advantages compared with biweekly oxaliplatin-regimens in terms of neurotoxicity.

In conclusion, weekly oxaliplatin plus 5-FU/FA has a favourable safety profile compared with previous data from studies of the biweekly FUFOX regimen. This weekly FUFOX regimen results in a high tumour response rate in first-line metastatic gastric cancer and is associated with a promising OS time. Therefore, the weekly FUFOX regimen can be recommended for phase III studies as well as a combination partner for new investigational drugs in phase I/II trials.

\section{ACKNOWLEDGEMENTS}

This study was supported by a grant from the Sanofi-Synthelabo $\mathrm{GmbH}$, Berlin, Germany. We thank Dr Matthias Suermondt and Dr Marco Schupp for their support. We also thank our research nurses Mrs Nadine Roethling and Mrs Brigitte Lang for their excellent work.

\section{REFERENCES}

Ajani JA, Baker J, Pisters PW, Ho L, Mansfield PF, Feig BW, Charnsangavej

C (2002) Irinotecan/cisplatin in advanced, treated gastric or gastro- esophageal junction carcinoma. Oncology (Huntington) 16(5 Suppl 5): $16-18$ 
Al-Batran SE, Atmaca A, Hegewisch-Becker S, Jaeger D, Hahnfeld S, Rummel MJ, Seipelt G, Rost A, Orth J, Knuth A, Jaeger E (2004) Phase II trial of biweekly infusional fluorouracil, folinic acid, and oxaliplatin in patients with advanced gastric cancer. J Clin Oncol 22: 658-663

André T, Boni C, Mounedji-Boudiaf L, Navarro M, Tabernero J, Hickish T, Topham C, Zaninelli M, Clingan P, Bridgewater J, Tabah-Fisch I, de Gramont A, Multicenter International Study of Oxaliplatin/5-Fluorouracil/Leucovorin in the Adjuvant Treatment of Colon Cancer (MOSAIC) Investigators (2004a) Oxaliplatin, fluorouracil, and leucovorin as adjuvant treatment for colon cancer. $N$ Engl J Med 350: 2343-2351

André T, Tournigand C, Rosmorduc O, Provent S, Maindrault-Goebel F, Avenin D, Selle F, Paye F, Hannoun L, Houry S, Gayet B, Lotz JP, de Gramont A, Louvet C, GERCOR Group (2004b) Gemcitabine combined with oxaliplatin (GEMOX) in advanced biliary tract adenocarcinoma: a GERCOR study. Ann Oncol 15: 1339-1343

Assersohn L, Brown G, Cunningham D, Ward C, Oates J, Waters JS, Hill ME, Norman AR (2004) Phase II study of irinotecan and 5-fluorouracil/ leucovorin in patients with primary refractory or relapsed advanced oesophageal and gastric carcinoma. Ann Oncol 15: 64-69

Caussanel JP, Levi F, Brienza S, Misset JL, Itzhaki M, Adam R, Milano G, Hecquet B, Mathe G (1990) Phase I trial of 5-day continuous venous infusion of oxaliplatin at circadian rhythm modulated rate compared with constant rate. J Natl Cancer Inst 82: 1046-1050

Chao Y, Yeh KH, Chang LT, Chao TY, Wu MF, Chang CS, Chang JY, Chung CY, Kao WY, Hsieh RK, Cheng AL (2004) Phase II study of weekly oxaliplatin and 24-h infusion of high-dose 5-fluorouracil and folinic acid in the treatment of advanced gastric cancer. Br J Cancer 91: 453-458

de Gramont A, Figer A, Seymour M, Homerin M, Hmissi A, Cassidy J, Boni C, Cortes-Funes H, Cervantes A, Freyer G, Papamichael D, Le Bail N, Louvet C, Hendler D, de Braud F, Wilson C, Morvan F, Bonetti A (2000) Leucovorin and fluorouracil with or without oxaliplatin as first-line treatment in advanced colorectal cancer. J Clin Oncol 18: 2938-2947

Ducreux M, Mitry E, Ould-Kaci M, Boige V, Seitz JF, Bugat R, Breau JL, Bouche O, Etienne PL, Tigaud JM, Morvan F, Cvitkovic E, Rougier P (2004) Randomized phase II study evaluating oxaliplatin alone, oxaliplatin combined with infusional 5-FU, and infusional 5-FU alone in advanced pancreatic carcinoma patients. Ann Oncol 15: 467-473

Freyer G, Bossard N, Romestaing P, Mornex F, Chapet O, Trillet-Lenoir V, Gerard JP (2001) Addition of oxaliplatin to continuous fluorouracil, l-folinic acid, and concomitant radiotherapy in rectal cancer: The Lyon $\mathrm{R}$ 97-03 phase I trial. J Clin Oncol 19: 2433-2438

Giacchetti S, Perpoint B, Zidani R, Le Bail N, Faggiuolo R, Focan C, Chollet P, Llory JF, Letourneau Y, Coudert B, Bertheaut-Cvitkovic F, LarregainFournier D, Le Rol A, Walter S, Adam R, Misset JL, Levi F (2000) Phase III multicenter randomized trial of oxaliplatin added to chronomodulated fluorouracil-leucovorin as first-line treatment of metastatic colorectal cancer. J Clin Oncol 18: $136-147$

Glimelius B, Ekstrom K, Hoffman K, Graf W, Sjoden PO, Haglund U, Svensson C, Enander LK, Linne T, Sellstrom H, Heuman R (1997) Randomized comparison between chemotherapy plus best supportive care with best supportive care in advanced gastric cancer. Ann Oncol 8: $163-168$

Goldberg RM, Sargent DJ, Morton RF, Fuchs CS, Ramanathan RK, Williamson SK, Findlay BP, Pitot HC, Alberts SR (2004) A randomized controlled trial of fluorouracil plus leucovorin, irinotecan and oxaliplatin combinations in patients with previously untreated metastatic colorectal cancer. J Clin Oncol 22: 23-30

Grothey A, Deschler B, Kroening H, Ridwelski K, Reichardt P, Kretzschmar A, Clemens M, Hirschmann W, Lorenz M, Asperger W, Buechele T, Schmoll HF (2002) Phase III study of bolus 5-fluorouracil (5-FU)/folinic acid (FA) (Mayo) vs weekly high-dose $24 \mathrm{~h} 5$-FU infusion/FA+oxaliplatin (OXA) in advanced colorectal cancer (ACRC). Proc Am Soc Clin Oncol 21: 129a (abstr 512)

Khushalani NI, Leichman CG, Proulx G, Nava H, Bodnar L, Klippenstein D, Litwin A, Smith J, Nava E, Pendyala L, Smith P, Greco W, Berdzik J, Douglass H, Leichman L (2002) Oxaliplatin in combination with protracted-infusion fluorouracil and radiation: report of a clinical trial for patients with esophageal cancer. J Clin Oncol 20: 2844-2850

Louvet C, Andre T, Lledo G, Hammel P, Bleiberg H, Bouleuc C, Gamelin E, Flesch M, Cvitkovic E, de Gramont A (2002b) Gemcitabine combined with oxaliplatin in advanced pancreatic adenocarcinoma: final results of a GERCOR multicenter phase II study. J Clin Oncol 20: 1512-1518

Louvet C, Andre T, Tigaud JM, Gamelin E, Douillard JY, Brunet R, Francois E, Jacob JH, Levoir D, Taamma A, Rougier P, Cvitkovic E, de Gramont A (2002a) Phase II study of oxaliplatin, fluorouracil, and folinic acid in locally advanced or metastatic gastric cancer patients. J Clin Oncol 20: $4543-4548$

Machin D, Campbell MJ (1997) Statistical Tables for the Design of Clinical Trials 2nd edn Oxford: Blackwell Scientific Publications, pp 281-282

Rödel C, Grabenbauer GG, Papadopoulos T, Hohenberger W, Schmoll HJ, Sauer R (2003) Phase I/II trial of capecitabine, oxaliplatin, and radiation for rectal cancer. J Clin Oncol 21: 3098-3104

Therasse P, Arbuck SG, Eisenhauer EA, Wanders J, Kaplan RS, Rubinstein L, Verweij J, Van Glabbeke M, van Oosterom AT, Christian MC, Gwyther SG (2000) New guidelines to evaluate the response to treatment in solid tumors. J Natl Cancer Inst 92: 205-216

Vanhoefer U, Rougier P, Wilke H, Ducreux MP, Lacave AJ, Van Cutsem E, Planker M, Santos JG, Piedbois P, Paillot B, Bodenstein H, Schmoll HJ, Bleiberg H, Nordlinger B, Couvreur ML, Baron B, Wils JA (2000) Final results of a randomized phase III trial of sequential high-dose methotrexate, fluorouracil, and doxorubucin versus etoposide, leucovorin, and fluorouracil versus infusional fluorouracil and cisplatin in advanced gastric cancer: A trial of the European Organization for Research and Treatment of Cancer Gastrointestinal Tract Cancer Cooperative Group. J Clin Oncol 18: 2648-2657

Waters JS, Norman A, Cunningham D, Scarffe JH, Webb A, Harper P, Joffe JK, Mackean M, Mansi J, Leahy M, Hill A, Oates J, Rao S, Nicolson M, Hickish T (1999) Long-term survival after epirubicin, cisplatin and fluorouracil for gastric cancer: results of a randomized trial. Br J Cancer 80: $269-272$ 